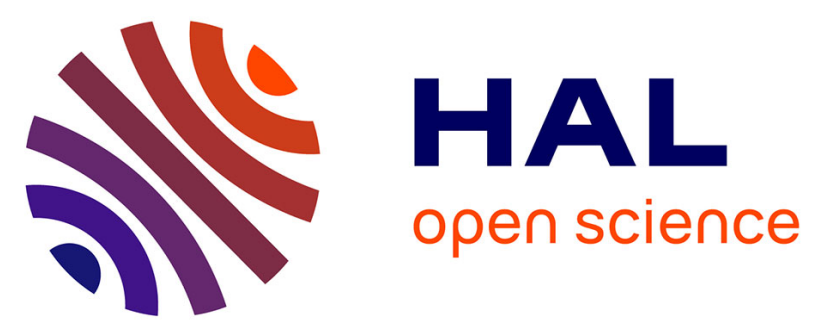

\title{
Generation and processing of urinary and plasmatic metabolomic fingerprints to reveal an illegal administration of recombinant equine growth hormone from LC-HRMS measurements
}

Fanny Kieken, Gaud Pinel, Jean-Philippe Antignac, Anne-Christelle Paris, Patrice Garcia, Marie-Agnès Popot, Morgane Grall, Victoria Mercadier, Pierre-Louis Toutain, Yves Bonnaire, et al.

\section{- To cite this version:}

Fanny Kieken, Gaud Pinel, Jean-Philippe Antignac, Anne-Christelle Paris, Patrice Garcia, et al.. Generation and processing of urinary and plasmatic metabolomic fingerprints to reveal an illegal administration of recombinant equine growth hormone from LC-HRMS measurements. Metabolomics, 2011, 7 (1), pp.84-93. 10.1007/s11306-010-0233-8 . hal-01191146v2

HAL Id: hal-01191146

https://hal.science/hal-01191146v2

Submitted on 2 Oct 2015

HAL is a multi-disciplinary open access archive for the deposit and dissemination of scientific research documents, whether they are published or not. The documents may come from teaching and research institutions in France or abroad, or from public or private research centers.

$$
\text { Copyright }
$$

L'archive ouverte pluridisciplinaire HAL, est destinée au dépôt et à la diffusion de documents scientifiques de niveau recherche, publiés ou non, émanant des établissements d'enseignement et de recherche français ou étrangers, des laboratoires publics ou privés. 


\title{
Generation and processing of urinary and plasmatic metabolomic fingerprints to reveal an illegal administration of recombinant equine growth hormone from LC-HRMS measurements
}

\author{
Fanny Kieken • Gaud Pinel • Jean-Philippe Antignac • Anne-Christelle Paris • \\ Patrice Garcia • Marie-Agnès Popot $\cdot$ Morgane Grall $\cdot$ Victoria Mercadier • \\ Pierre Louis Toutain · Yves Bonnaire $\cdot$ Bruno Le Bizec
}

Received: 1 March 2010/Accepted: 14 August 2010/Published online: 2 September 2010

(C) Springer Science+Business Media, LLC 2010

\begin{abstract}
Growth hormones are proteins produced by the anterior pituitary gland responsible for bone and tissue growth through their effects on carbohydrates, lipids and proteins metabolisms. Despite strict regulations banning the use of recombinant equine growth hormone, this substance is suspected to be misused to improve the horse physical performances. In order to check whether the regulation is fulfilled or not, controls are organized and a new analytical screening tool potentially able to detect such abuse was investigated in this paper. An untargeted metabolomics approach, based on liquid chromatography coupled to high resolution mass spectrometry, was developed and applied to characterize and compare horse urinary and plasmatic metabolic profiles upon reGH administrations. After minimal sample preparation, biological fluids were analyzed by LC-ESI $( \pm)$-Q-TOF. Data processing was performed by XCMS software and multivariate data analysis applied to the generated data set allowed building OPLS models to discriminate control versus treated populations. Results demonstrated significant metabolic modifications consecutively to the reGH treatment. A
\end{abstract}

F. Kieken · G. Pinel $(\bowtie) \cdot$ J.-P. Antignac · B. Le Bizec Laboratoire d'Etude des Résidus et Contaminants dans les Aliments (LABERCA)USC INRA 2013, ONIRIS, Route de Gachet, BP 50707, 44307 Nantes Cedex 3, France

e-mail: laberca@oniris-nantes.fr

F. Kieken - A.-C. Paris · P. Garcia - M.-A. Popot - Y. Bonnaire LCH Laboratoire des Courses Hippiques, 15 Rue de Paradis, 91370 Verrières-le-Buisson, France

M. Grall · V. Mercadier · P. L. Toutain

UMR 181 Physiopathologie et Toxicologie Expérimentales

INRA, Toulouse, France comparative study between urinary and plasmatic signatures was performed to evaluate the resulting metabolomic models and to asses their respective interests in the scope of real application for screening reGH administration.

Keywords Growth hormone - Horse racing . High resolution mass spectrometry $\cdot$ Metabolomics

$\begin{array}{ll}\text { Abbreviations } \\ \text { GH } & \text { Growth hormone } \\ \text { reGH } & \text { Recombinant equine growth hormone } \\ \text { IGF-I } & \text { Insulin like growth factor-I } \\ \text { LC } & \text { Liquid chromatography } \\ \text { ESI } & \text { Electrospray ionization } \\ \text { MS } & \text { Mass spectrometry }\end{array}$

\section{Introduction}

Growth hormones (GH), also named somatotropins (ST), are around 190 amino acids polypeptides produced by the anterior pituitary gland of mammals (Rahmanian et al. 1997). If GH is primarily responsible for bone and tissue growth, this molecule exhibits also many other biological effects associated with developmental and reproductive functions (Thompson 1997; Etherton and Bauman 1998). For health safety and economical reasons, available growth hormones are nowadays recombinant molecules. Recombinant equine growth hormone (reGH) was first marketed in Australia (EquiGen $-5^{\circledR}$ ) in order to improve the positive nitrogen balance in horses over 15 years (Malinowski et al. 1997). However, it is also suspected to be diverted from its original purpose to improve physical performance and 
wound healing (Dart et al. 2003). In this context, the racing rules have strictly prohibited the use of recombinant equine growth hormone in France. In order to check whether the regulation is fulfilled or not, controls have been organized and efficient methods based on mass spectrometry detection have been set up. To date, the screening of reGH administration focuses on specific biomarkers such as Insulin-like growth factor-1 or antibodies anti-reGH (Popot et al. 2001;(Bailly-Chouriberry 2008a) whereas confirmation methods relies on LC-MS/MS measurement of the reGH itself in plasma (Bailly-Chouriberry 2008b).

Despite their efficiency, the current screening strategies for controlling their potential illegal use face some limits such as the establishment of biomarkers thresholds or the delay necessary before inducing an immunological response, which are both animal dependant. Since growth hormone acts on many action sites via carbohydrate and lipidic metabolisms (Szeszycki et al. 1996), a wide range of potential biomarkers could be expected to be monitored for such screening analysis. In this context, metabolomics may represent a new emerging approach for investigating the global physiological effects associated to the administration of growth hormone and therefore constitute an alternative screening strategy.

Metabolomics, also known as global metabolomic profiling, basically consists in the untargeted study of relatively polar and low molecular weight compounds (typically $<$ $1500 \mathrm{Da})$ present in biological samples and accessible to the analysis (Nicholson et al. 2002). Since these so-called metabolites are the end-products of cellular regulatory processes, and since their levels can be regarded as the ultimate response of biological systems to any changes, studying the metabolome through biological fluids (metabolic profiling or fingerprinting) is expected to reveal potential metabolomic disruption and therefore highlight relevant biomarkers of exposure or effect associated to drug administration. Two main analytical techniques are predominantly reported and described in metabolomics studies: 1H nuclear magnetic resonance spectroscopy (NMR) (Nicholson et al. 1999) and gaz or high-performance liquid chromatography coupled to mass spectrometry (Bajad and Shulaev 2007; Theodoris et al. 2008). NMR which has traditionally been used in this context present clear advantages in terms of robustness and reproducibility but MS based methods have recently proved to be valuable for such studies, especially thanks to recent technological advances (Tolstikov and Fiehn 2002; Tolstikov et al. 2003; Idborg et al. 2005) and furthermore present some incomparable advantages over NMR either in terms of sensitivity or capabilities for structural elucidation purposes. Several ionization/desorption techniques have been used in MS metabolomics, including FAB (Platt et al. 1988), MALDI (Jarman et al. 2000) or Electrospray (McDougall et al.
2008). High resolution mass analysers such as time of flight (TOF) (Kind et al. 2007), Fourier transform ion cyclotron resonance (Sleighter and Hatcher 2007) and the recently introduced LTQ-Orbitrap analysers (Dunn et al. 2008) then remains the instruments of choice in this field.

The first metabolomics studies were originally and primarily focused on clinical (Kind et al. 2007; Lewis et al. 2008) or pharmaceutical applications (Robertson 2005) but new more specific areas of application have recently emerged, for instance in the anti-doping field. In this specific domain, metabolic variations induced by physiological response to hormonal treatment have already been investigated and evidenced using NMR (Dumas 2002a, b). These two particular studies were the first to demonstrate the suitability of a metabolomics strategy as a powerful tool to screen for anabolic steroid administration in cattle. More recently, a study based on LC-HRMS urinary fingerprints demonstrated the relevance of such an untargeted metabolomics approach to discriminate control versus clenbuterol treated calves (Courant et al. 2009). Regarding the growth hormone issue, a similar study was successfully reported to highlight metabolomic perturbations associated with reGH treatment (Kieken et al. 2009) through LC-HRMS urinary biological signatures. This preliminary study aimed at assessing the feasibility of such an approach in the context of growth hormone abuse in horses, was however conducted on a relatively reduced number of animals. In addition, if urine is often reported as a matrix of interest in such studies since it is a fluid of excretion containing a large number of potential interesting metabolites which can easily be collected (Courant et al. 2009; Kieken et al. 2009; Rijk et al. 2009), the use of plasma is also reported in many applications and has demonstrated its relevance in the discovery of new biomarkers (Giovane et al. 2008; Liu et al. 2009).

In this context, the aim of the present work was to generate and compare plasmatic LC-HRMS fingerprints of low molecular weight metabolites collected from control and GH-treated horses to assess the global biological effect consecutive to reGH-treatment by means of chemometric models. Analyses were conducted either in positive and negative ionization electrospray mode in order to investigate different sub-fraction of the metabolome. Finally, a urine profiling study was also performed to compare and assess the two respective chemometrics models, and then the relevancies of the two biological matrices.

\section{Materials and methods}

\subsection{Chemicals and reagents}

Recombinant equine growth hormone, reGH (EquiGen- $5^{\circledR}$ ) was purchased from BresaGen Ltd (Thebarton, Australia). 
HPLC grade acetonitrile (MeCN), acetic acid, and ammonium acetate were purchased from Carlo Erba Reactifs (SDS, Peypin, France). d3-testosterone and d3-epi-testosterone-glucuronide were obtained from LGC Standards (Molsheim, France). Deionised water was produced using an ultra pure water system (Milli-Q, Millipore Corporation, Billerica,USA).

\subsection{Animals, treatment and sample preparation}

Experimentation was led in agreement with animal welfare rules at the National Veterinary School of Toulouse (France). Thirteen standarbred horses $(5$ mares and 8 geldings) weighting from 430 to $610 \mathrm{~kg}$ and aged between 4 and 20 years-old have been used and divided in two groups, assigned as control and treated animals. Administrated horses received reGH by subcutaneous injection at $18 \mu \mathrm{g} \mathrm{kg}^{-1}$ during 14 days and the control group was administrated with the excipient only. In both groups, plasma and urine samples were collected daily during the week preceding the first injection (i.e. days $\mathrm{D}_{-7}$ to $\mathrm{D}_{-1}$ ) and during the three-first weeks after the first injection (i.e. days $D_{+1}$ to $D_{+20}$ ). Samples were then collected every 2 days until $\mathrm{D}_{+69}$. Samples were stored at $-20^{\circ} \mathrm{C}$ until analysis. ReGH doses were chosen according to the recommendations of the manufacturer.

\subsection{Sample preparation}

The sample preparation was based on the filtration of biological samples (plasma and urine) through a $10 \mathrm{kDa}$ polyethersulfone membrane (Microsep $10 \mathrm{~K}$ Omega, VWR, Fontenay sous bois, France) under centrifugation at $4000 \mathrm{rpm}$ at $5^{\circ} \mathrm{C}$ for $30 \mathrm{~min}$. The filtered samples were then frozen at $-20^{\circ} \mathrm{C}$. Before LC-HRMS analyses, $5 \mu \mathrm{l}$ of a solution containing two internal standards (d3-testosterone and $\mathrm{d} 3$-epi-testosterone-glucuronide), both at $3 \mathrm{ng} \mathrm{\mu l}^{-1}$, were added to $20 \mu \mathrm{l}$ of each sample. The mixture was shaken and $5 \mu \mathrm{l}$ of this solution were injected on the LC system.

\subsection{LC-HRMS analysis}

\subsubsection{Liquid chromatography}

Chromatographic separation was performed with an Ultimate 3000 (Dionex, Sunnyvale, USA) pump on a reversed phase $(2.1 \mathrm{~mm} \times 100 \mathrm{~mm}$ Uptisphere Strategy NEC, $2.2 \mu \mathrm{m}$ particle size, Interchim, Montluçon, France). The analytes were eluted by a 20 min gradient, started at $100 \%$ A (Water $+0.1 \%$ acetic acid) to $100 \% \mathrm{~B}$ (Acetonitrile $+0.1 \%$ acetic acid) in 13 min, was maintained at $100 \%$ $\mathrm{B}$ during $1 \mathrm{~min}$ and then returned to the initial condition within $4 \mathrm{~min}$. The chromatographic system was further maintained at 100\% A during 2 min (equilibration time).

\subsubsection{ESI-HRMS}

High-resolution MS fingerprints were acquired on a hybrid mass spectrometer (MicroToF Q-II, Bruker, Bremen, Germany) in both positive and negative electrospray ionization mode. The capillary voltage was set at $5 \mathrm{kV}$. The other spectrometric parameters were set as follows: capillary temperature: $180^{\circ} \mathrm{C}$, nebulizer "gas flow": 2.4 bar, dry "gaz flow": $8 \mathrm{~L} \mathrm{~min}{ }^{-1}$. A solution of sodium cluster was used for external calibration of the instrument. For analyses performed in negative mode, the value of capillary voltage was changed and set at $3.5 \mathrm{kV}$, the other parameters remained unchanged. Centroid full scan mass spectra were acquired in the [200-1500] and [50-800] mass ranges for plasma and urine samples analysis, respectively.

\subsection{Data processing}

After LC-HRMS analysis, the original data files were converted to the NetCDF format (.cdf) for further data processing using a conversion software program (file converter program available from Bruker Daltonics Data Analysis software).

The data processing was achieved using XCMS (Smith et al. 2006) software implemented with the $\mathrm{R}$ statistical language, as described elsewhere (Kieken et al. 2009). Briefly, the process is based on several steps: peak picking, peak grouping, retention time alignment followed by a first statistical test which is performed on the whole data set to calculate the $\mathrm{P}$ value and the fold change associated to each detected MS signal. This step is based on the comparison of the MS signal abundances of extracted ions present in both control and GH-treated populations. Finally, the signals are attributed a rank according to their $\mathrm{P}$ value.

\subsection{Data analysis}

As previously described (Kieken et al. 2009) SIMCA$\mathrm{P}+$ (v 12.0, Umetrics, Sweden) software was used for multivariate data analysis. Orthogonal Partial Least Square (OPLS) analyses were applied to build descriptive and predictive models. These OPLS models attempt to explain and predict one $Y$ variable (in our study the status of the animals involved, i.e. GH-treated or Control) from a $X$ matrix constituted by all the ions constituting the fingerprints. The two main components generated by the analysis are as follows: axis $1(=[\mathrm{t} 1])$ is a linear combination of ions selected after data processing, it aims at better explaining the discrimination between $\mathrm{GH}$-treated and control animals; axis $2(=[\mathrm{t} 2])$, orthogonal to axis 1 , is 
a linear combination of the ions detected during data processing which aims at restoring the residual variability. The validity and robustness of models were evaluated by $\mathrm{R} 2(Y)$ and $\mathrm{Q} 2(Y)$ parameters, cross validation, permutation test and CV-ANOVA.

\section{Results and discussion}

\subsection{Quality of LC-HRMS data}

If in conventional target compounds analysis, the quality of the data produced are controlled by the incorporation of internal standards and specific quality control samples (QCs) which are often blank samples spiked with known concentrations of the target analytes, this kind of approach is much more difficult in metabonomics analysis where the samples typically contain $100 \mathrm{~s}-1000 \mathrm{~s}$ of components covering a wide range of concentrations and structural types, of variable and unknown MS responses. It is however important to control the quality of the data produced; therefore several quality controls have been introduced in the present study. Pools of samples were injected at the beginning, the end but also randomly all along the analytical run in order to check for consistency during analysis. In addition, two "internal" standards (d3-testosterone and d3-epi-testosterone-glucuronide) have been added to each sample before LC-HRMS analysis in order to evaluate the retention time stability, the consistency of signal intensities and mass accuracy within and between measurement series. No significant drift in retention time was observed within and between measurement series; the deviation from standard accurate masses remained below $5 \mathrm{ppm}$.

\subsection{Investigation of plasma metabolome under ESI+}

Ninety-six plasma samples were filtered under $10 \mathrm{kDa}$ cutoff membranes, which is a sample preparation already assessed in previous works regarding its relevance for metabolomics study (Courant et al. 2009; Kieken et al. 2009). The samples were then analyzed by LC-HRMS in positive electrospray ionization. Centroid full scan mass spectra were acquired in the [200-1500] mass range, adapted to the analysis of soluble substances present in the plasma such as carbohydrates, lipids or small proteins. The choice of a chromatographic separation step was naturally imposed given the complexity of the plasma matrix. However, phase particles of small size $(2.2 \mu \mathrm{m})$ were used to reduce the length of the analysis as compared to the protocol presented in a previous study (Kieken et al. 2009). LC-HRMS data were then processed by XCMS software and 5086 ions exhibiting signal-to-noise ratio better than 6 were reported as constituting the acquired metabolic fingerprints. A supervised multivariate analysis OPLS (Orthogonal Partial Least Square) was then performed on the basis of all acquired metabolic fingerprints in order to set up a descriptive model. The resulting model (Fig. 1a) clearly shows that plasma samples collected from control animals $(\boldsymbol{\Delta} 0)$ have coordinates opposed to plasma samples collected from GH-treated horses $(\boldsymbol{\Delta} 1)$ which leads to their good visual discrimination on the figure. These good performances were confirmed by the high values found for $\mathrm{R}^{2}(Y)=0.85$ and $\mathrm{Q}^{2}(Y)=0.50$.

In the scope of targeting candidate biomarkers of reGH administration, a selection of ions more particularly involved in the discrimination of control and GH-treated populations was performed from the OPLS model presented in Fig. 1a. First, the so-called "S-plot" associated to this model was examined to visualize the contribution of each ion to the model and thus highlight which of them were the most correlated to the first discriminant component (Fig. 2). A particular attention was paid to those ions and furthermore, only those exhibiting $\mathrm{P}$ value lower than 0.05 and fold change higher than 1.5 were selected. Finally, a visual examination of the XCMS overlaid extracted ions chromatograms was completed in order to eliminate any potential artefacts MS signals. The successive application of all these criteria finally led to the selection of 56 ions.

A new OPLS analysis was then performed on the basis of these 56 selected ions which shows the possibility to still separate plasma collected from control $(\boldsymbol{\Delta} 0)$ or GH-treated animals $(\mathbf{\Delta} 1)$ with acceptable descriptive $\left(\mathrm{R}^{2}(Y)=0.7\right)$ and predictive $\left(\mathrm{Q}^{2}(Y)=0.5\right)$ capabilities (Fig.1b). Only samples collected from treated animals 69 days after the first reGH administration cannot be discriminated from control population (black circle) which may be explained by the fact that metabolic perturbations associated to reGH administration are no longer detectable on the basis of 56 selected ions. The robustness of this model was further investigated by several steps. First, a cross-validation was performed. It consists to build a new OPLS analysis on the basis of $2 / 3$ of the original dataset. The other $1 / 3$ of the dataset is considered as validation test and is incremented in the model for the prediction. The results (Fig. 3 a) are satisfactory since they show the possibility to build a reliable descriptive model $\left(\mathrm{R}^{2}(Y)=0.78 \mathrm{Q}^{2}(Y)=0.51\right)$ where control $(\mathbf{\Delta} 0)$ and $\mathrm{GH}$-treated $(\mathbf{\Delta} 1)$ populations are discriminated. Moreover, the prediction of the remaining data composed of control animals ( $\left.\boldsymbol{\Xi}_{2}\right)$ and $\mathrm{GH}$-treated animals ( 3 ) allowed the classification of these samples without misallocation. The cross-validation thus demonstrates that the model could be used as a future predictive tool for the classification of unknown samples. Nevertheless, a permutation test must be absolutely performed to ensure that these results are not due to a chance factor. It consists in reallocating randomly the status of the animals 
Fig. 1 OPLS analyses of plasma samples collected from control and reGH-treated horses. $\boldsymbol{\Delta} 0$ coordinates of samples from control animals (D-7 to $D_{-1}$ ); $\mathbf{\Delta} 1$ coordinates of samples from GH-treated animals $\left(\mathrm{D}_{+1}\right.$ to $\left.\mathrm{D}_{+69}\right)$. Analyses were performed in positive ionization mode with mass range from 200 to $1500 \mathrm{~m} / \mathrm{z}$. The models are based on 5086 ions (a) and 56 ions (b), respectively. The ions are present with different MS abundance in the plasma collected at $\mathrm{D}_{-7}$ to $\mathrm{D}_{-1}$, and $\mathrm{D}_{+1}$ to $\mathrm{D}_{+69}$
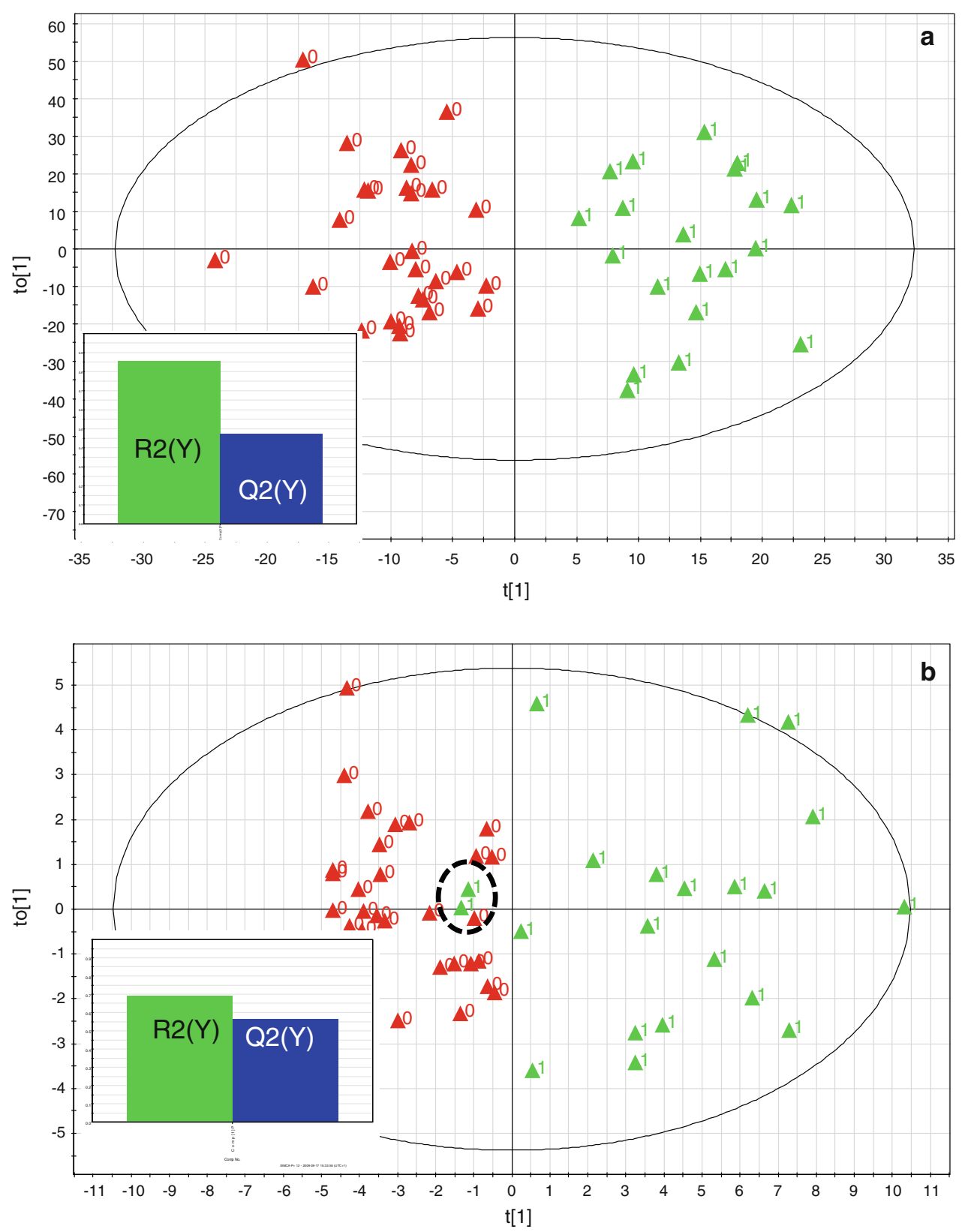

(i.e. control or GH-treated). Figure $3 b$ shows that $\mathrm{R} 2(Y)$ and Q2 $(Y)$ values decrease upon permutations which demonstrate that the model is not the result of a chance factor but a valid and robust discrimination between control and reGH-treated populations. Finally a CV-ANOVA (Cross Validation-ANaysis Of Variance) was calculated. It allows attributing a degree of significance to the test-permutation. The results of CV-ANOVA variant suggest that the model is significant with a $\mathrm{P}$ value of 0.004 .

\subsection{Investigation of plasma metabolome under ESI-}

Plasma samples were also analyzed after negative electrospray ionization mode but maintaining all other acquisition parameters identical. During the data processing step, 3039 ions constituting the fingerprints were extracted by XCMS and further subjected to multivariate statistical analyses (Fig. 4a). An efficient discrimination was observed between control and GH-treated animals with high $\mathrm{R}^{2}(Y)=0.85$ and $\mathrm{Q}^{2}(Y)=0.7$ values. A focus on the ions the most involved in the discrimination of control $(\boldsymbol{\Delta} 0)$ and GH-treated animals $(\boldsymbol{\Delta} 1)$ was carried out. In the same manner as for the analysis conducted in positive ionization mode, only ions strongly correlated to the first discriminant component, and presenting $\mathrm{P}$ value lower than 0.05 as well as fold change higher than 1.5 were selected as ions of interest. These criteria enabled to highlight 86 ions as relevant from a statistical point of view. 


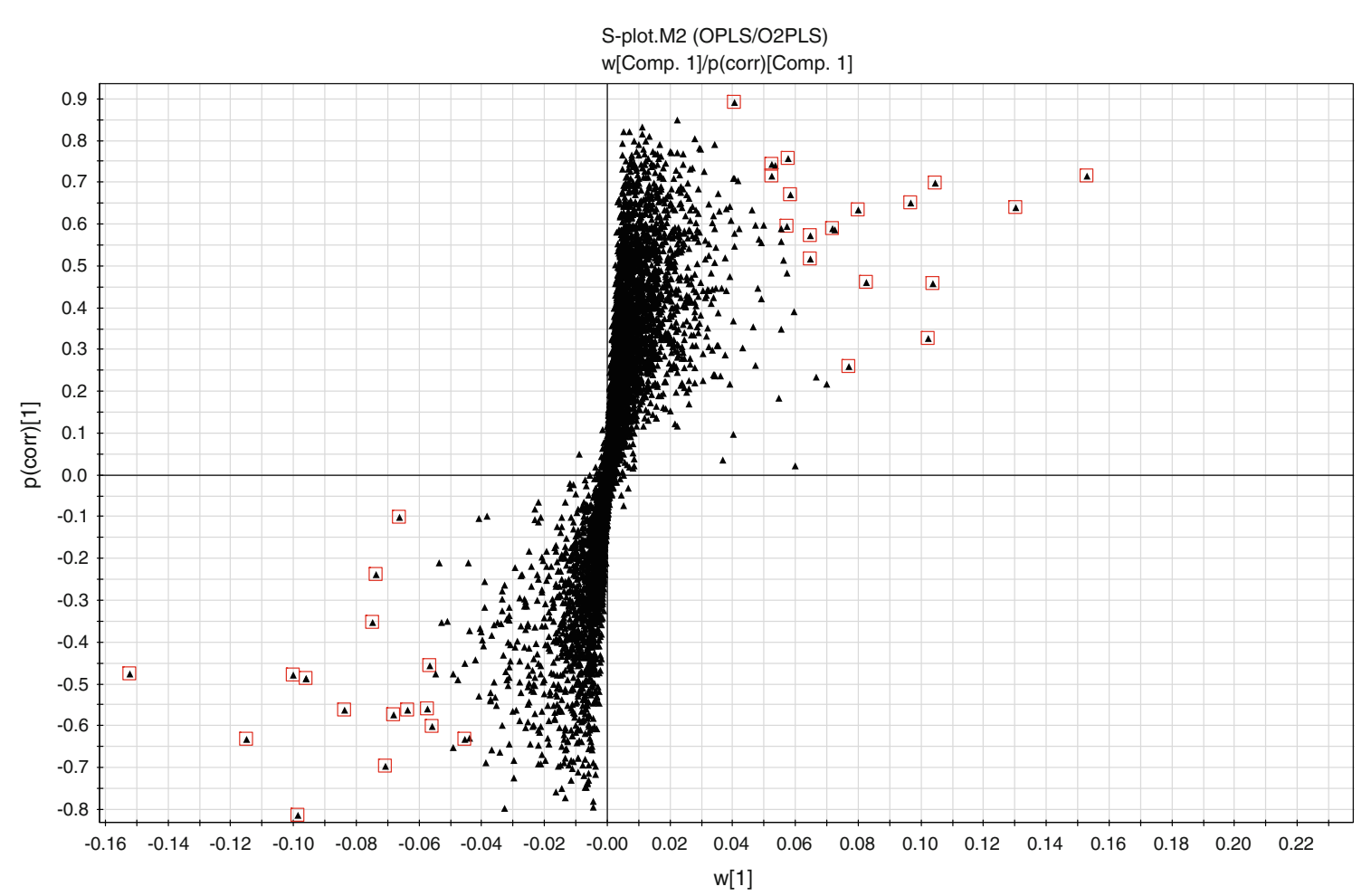

Fig. 2 S-Plot corresponding to OPLS analysis presented in Fig. 1a

An OPLS was further performed on these 86 ions and is presented in Fig. 4b. It reveals an efficient separation between the samples collected on control $(\mathbf{\Delta} 0)$ and $\mathrm{GH}$ treated $(\mathbf{\Delta} 1)$ animals. Besides these satisfactory results obtained in the negative ionization mode, it should be specified that, and as a general rule, all the ions highlighted during this study presented very low signal intensities which would impose a preliminary step of concentration of the samples before any further structural elucidation. A cross-validation and test-permutation were performed (data not shown) which allowed to definitively validate the model.

A thorough examination of all candidate ions finally selected in ESI + and ESI- revealed that only three ions were common to both ionization modes. The clear majority of these potential MS signal of interest ranged from 200 to $1000 \mathrm{~m} / \mathrm{z}$, indicating that the developed analytical strategy is as expected many suitable for detecting metabolites with relatively low molecular weights. However, few retained ions (observed in positive ion mode) presented $\mathrm{m} / \mathrm{z}$ ratio above 1000. Unsurprisingly, these results globally confirmed that potential biomarkers detected in positive and negative ionization mode are largely complementary. Consequently, the combination of both techniques would allow the access to a wider range of metabolites. Last, the chromatographic behavior observed for these potential metabolites of interest indicated they have very polar to relatively polar physico-chemical properties.

\subsection{Comparison of metabolomic models developed on urine and plasma matrices}

Urine samples from the same animals were also analyzed using the same analytical workflow but in positive ionization mode only (Fig. 1) as described in a previous work (Kieken et al. 2009). The LC-HRMS data were processed by XCMS software which allowed the detection of 5980 ions. The objective was to build an efficient and practically operational predictive model of reGH administration and then, to focus on a limited number of more specifically relevant variables. The same strategy as performed on plasma samples was then also applied on these urine samples [i.e. OPLS analysis, S-plot examination, application of statistical criteria for selection $(\mathrm{P}$ value $<0.05$, ratio $>11.51$ )]. Finally, 80 ions exhibiting MS intensities responsible for differences observed between both populations were targeted among the 5980 ions constituting the original metabolomic fingerprints (Fig. 5a). In the same manner as analyses performed on plasma matrix, the model was subjected to a validation. The test-permutation showed in Fig. 5b reveals that $\mathrm{R} 2(Y)$ and $\mathrm{Q} 2(Y)$ decreases upon permutation which demonstrates the model validity. A 
Fig. 3 Validation of dataset presented in Fig. 1b by crossvalidation and test-permutation. For the cross-validation (a), the dataset is divided as follows: $2 / 3$ of data for the building of descriptive model ( $\boldsymbol{\Delta} 0$ control and $1 \mathrm{GH}$-treated coordinates) populations; $\boldsymbol{\Delta} 1 / 3$ data for the prediction ( 2 control and $\boldsymbol{\square}_{3}$ GH-treated coordinates). For the test-permutation (b), the status of animals (i.e. control or $\mathrm{GH}$ treated) controlled by $Y$ variable is randomly reallocated
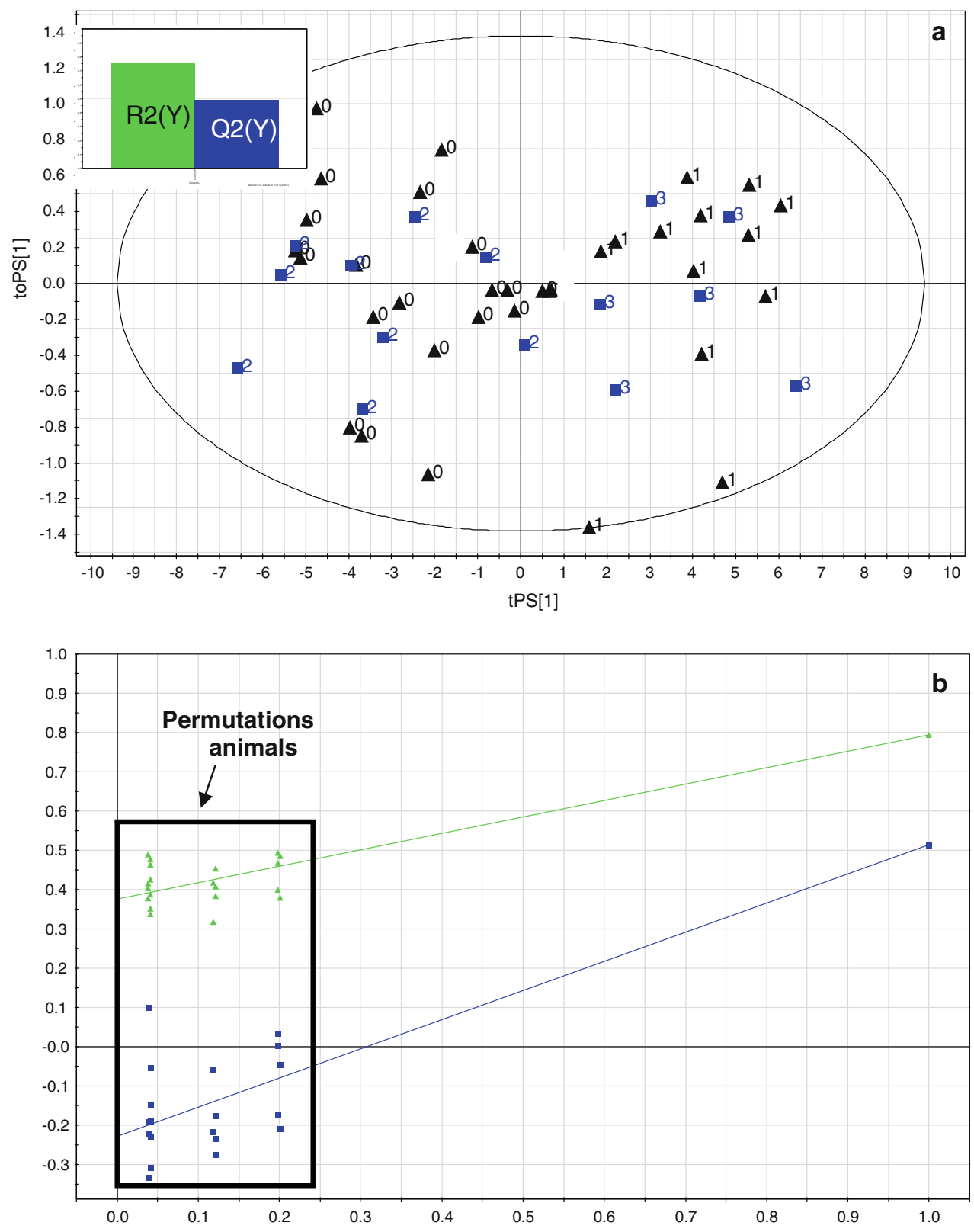

comparison of the models obtained after ESI+ fingerprinting of urine and plasma samples lead to the conclusion that the two biological matrices were equivalently descriptive since similar values of $\mathrm{R} 2(Y)$ were observed; however, the predictive ability of the models was found higher for plasma as attested by a higher $\mathrm{Q} 2(Y)$ value. This significant higher value of $\mathrm{Q} 2(Y)$ in the plasma model may be due to the fact that this particular matrix is less subjected to large variations in its composition than urine. Unlike plasma and most biological fluids, which exhibit tightly controlled solutes concentrations, urine composition is known to vary widely based upon water consumption and other physiological factors (Warrack et al. 2009).
However, urine metabolomic model was found interesting for its possibility to discriminate samples collected 69 days after the first reGH administration from samples collected on treated population which emphasize the fact that different metabolomes are investigated in urine and plasma. In this context, urine seems to be more interesting for a long-term screening of reGH abuse in horses. No common ion of interest could be detected between urine and plasma models, demonstrating that the nature of analyzed compounds is different according to biological matrix. Metabolites present in urine and plasma certainly exhibit different physicochemical properties which explains why urine matrix is more interesting for a long-term control. 
Fig. 4 OPLS plasma samples collected from control and reGH-treated horses. $\Delta 0$ coordinates of samples from control animals ( $\mathrm{D}_{-7}$ to $\mathrm{D}_{-1}$ );

$\Delta 1$ coordinates of samples from GH-treated animals $\left(\mathrm{D}_{+1}\right.$ to $\left.\mathrm{D}_{+69}\right)$. Analyses were performed in negative ionization mode with mass range from 200 to $1500 \mathrm{~m} / \mathrm{z}$. OPLS models are based on 3039 ions (a) and 86 ions (b), respectively
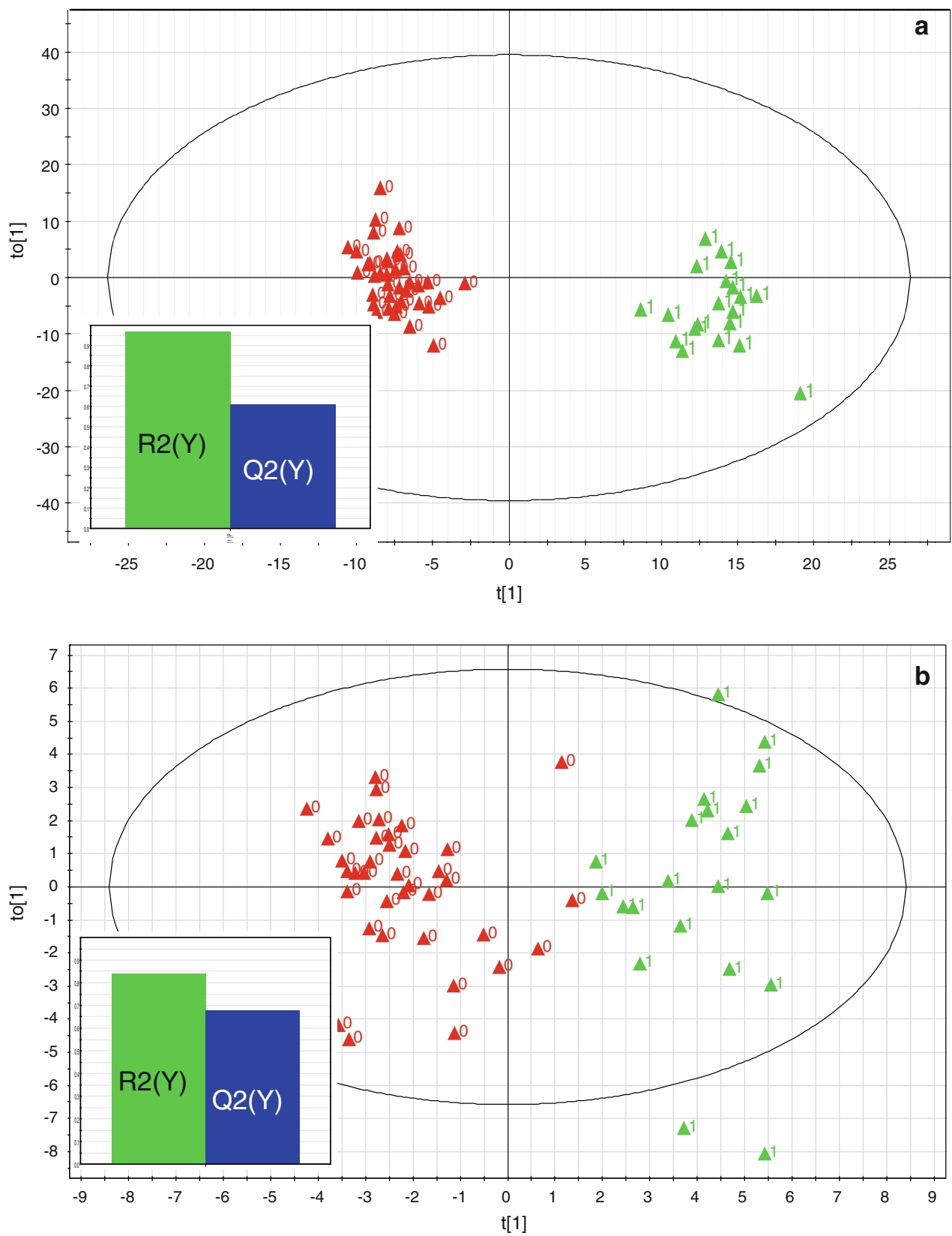

\section{Conclusions}

LC-HRMS fingerprinting in positive and negative ionization mode has been applied to plasma samples for the assessment of the effect of reGH administration on horse metabolism (without identifying any specific metabolites in the profile). The comprehensive metabolomics approach described in this paper enables to examine small molecules up to $1500 \mathrm{Da}$ in plasma samples. Through chemometric models, modifications of horse metabolism upon reGH administration were highlighted thanks to fingerprinting analyses acquired in both ionization modes. Relevant potential candidate biomarkers have been selected and have proved their ability to discriminate control from reGH-treated animals. However, the low MS intensity of ions targeted in negative mode may be problematic for further structural elucidation step where the abundance of metabolites is a crucial criterion to obtain satisfying mass precisions on molecular ion and its fragments. Regarding analysis performed in the positive ionization mode, the concentration of metabolites would be sufficiently abundant to consider a chemical identification without the development of a previous purification step. Plasmatic and urine biological signatures were compared and revealed dissimilarities and complementarities. Indeed, no common ion of interest could be highlighted between the both 
Fig. 5 OPLS analysis of urine samples collected from control and reGH-treated horses (a). $\mathbf{\Delta} 0$ coordinates of samples from control animals $\left(\mathrm{D}_{-7}\right.$ to $\left.\mathrm{D}_{-1}\right)$;

$\Delta 1$ coordinates of samples from GH-treated animals $\left(\mathrm{D}_{+1}\right.$ to $\mathrm{D}_{+69}$ ). Analysis was performed in positive ionization mode with mass range from 50 to $800 \mathrm{~m} / \mathrm{z}$. The model is based on 80 variables (MS signals with given retention time and $\mathrm{m} / \mathrm{z}$ value). For the test-permutation (b), the status of animals (i.e. control or GH-treated) controlled by $Y$ variable is randomly reallocated
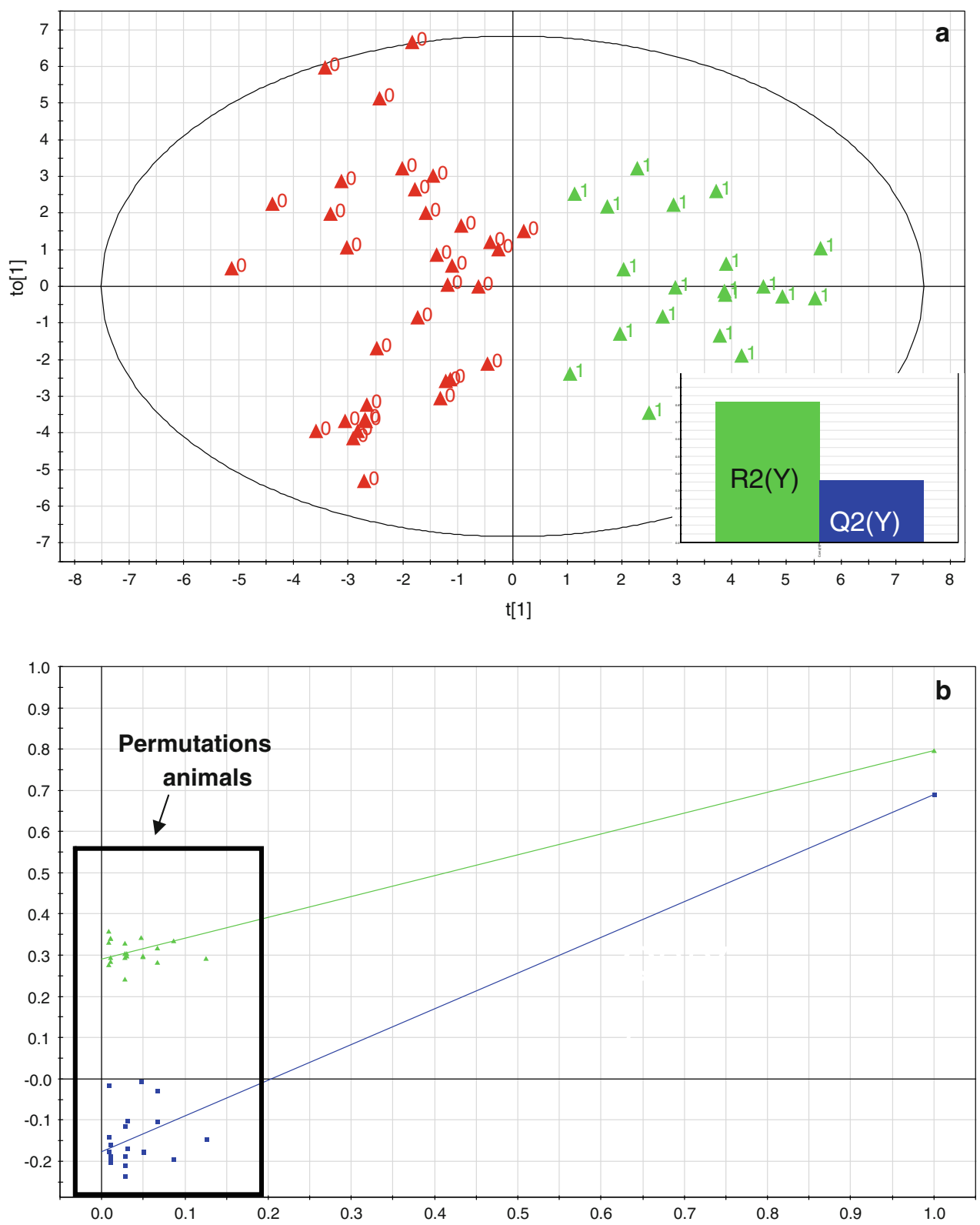

models, demonstrating that metabolic pathways are not the same in urine and plasma. However, plasma proved to be interesting for the prediction of unknown sample and urine more promising for long term detection. Urine and plasma can be seen as essential matrices for the development of metabolomics approach applied to the control of reGH administration.

Acknowledgment This work was supported by the French "Association Nationale de la Recherche et de la Technologie" (ANRT).

\section{References}

Bailly-Chouriberry, L., Pinel, G., Garcia, P., Popot, M. A., Bonnaire, Y., \& Lebizec, B. (2008a). Detection of secondary biomarker of
met-eGH as a strategy to screen for somatotropin misuse in horseracing. Analyst, 133, 270-276.

Bailly-Chouriberry, L., Pinel, G., Garcia, P., Popot, M. A., Bonnaire, Y., \& Lebizec, B. (2008b). Identification of recombinant equine growth hormone in horse plasma by LC-MS/MS: a confirmatory analysis in doping control. Analytical Chemistry, 80, 8340-8347.

Bajad, S., \& Shulaev, V. (2007). High-parallel metabolomics approaches using LC-MS2 for pharmaceutical and environmental analysis. Trends in Analytical Chemistry, 26, 625-636.

Courant, F., Pinel, G., Bichon, E., Monteau, F., Antignac, J. P., \& Le Bizec, B. (2009). Development of a metabolomic approach based on liquid chromatography-high resolution mass spectrometry to screen for clenbuterol abuse in calves. Analyst, 134, $1637-1646$.

Dart, A. J., Little, C. B., Hughes, C. E., Chu, Q., Dowling, B. A., Hodgson, D. R., et al. (2003). Recombinant equine growth hormone administration: Effects on synovial fluid biomarkers and cartilage metabolism in horses. Equine Veterinary Journal, $35,302-307$. 
Dumas, M. E., Canlet, C., André, F., Vercauteren, J., \& Paris, A. (2002a). Analyzing the physiological signature of anabolics steroids in cattle urine using pyrolysis/metastable atom bombardment mass spectrometry and pattern recognition. Analytical Chemistry, 74, 5393-5404.

Dumas, M. E., Debrauwer, L., Beyet, L., Lesage, D., Andre, F., Paris, A., et al. (2002b). Assessment of physiological disruptions using 1H-13C HMBC-NMR spectroscopy combined with pattern recognition procedures performed on filtered variables. Analytical Chemistry, 74, 2261-2273.

Dunn, W. B., Broadhurst, D., Brown, M., Baker, P. N., Redman, C. W. G., Kenny, L. C., et al. (2008). Metabolic profiling of serum using ultra performance liquid chromatography and the LTQOrbitrap mass spectrometry system. Journal of Chromatography B, 871, 288-298.

Etherton, T. D., \& Bauman, D. E. (1998). Biology of Somatotropin in Growth and Lactation of Domestic Animals. Physiological Reviews, 78, 745-761.

Giovane, A., Balestrieri, A., \& Napoli, C. (2008). New insights into cardiovascular and lipid metabolomics. Journal of Cellular Biochemistry, 105, 648-654.

Idborg, H., Zamani, L., Edlund, P. O., Schuppe-Koistinen, I., \& Jacobsson, S. P. (2005). Analysis by hydrophilic interaction liquid chromatography-electrospray ionization mass spectrometry. Journal of Chromatography analytical technologies in the biomedical and life sciences, 828, 9-13.

Jarman, K. H., Cebula, S. T., Saenz, A. J., Petersen, C. E., Valentine, N. B., Kingsley, M. T., et al. (2000). An algorithm for automated bacterial identification using matrix-assisted laser desorption/ ionization mass spectrometry. Analytical Chemistry, 72, $1217-1223$.

Kieken, F., Pinel, G., Antignac, J. P., Monteau, F., Paris, A. C., Popot, M. A., et al. (2009). Development of a metabonomic approach based on LC-ESI-HRMS measurements for profiling of metabolic changes induced by recombinant equine growth hormone in horse urine. Analytical Bioanalytical Chemistry, 8, 2119-2128.

Kind, T., Tolstikov, V., Fiehn, O., \& Weiss, R. (2007). A comprehensive urinary metabolomic approach for identifying kidney cancer. Analytical Biochemistry, 363, 185-195.

Lewis, G. D., Asnani, A., \& Gerszten, R. E. (2008). Application of metabolomics to cardiovascular biomarker and pathway discovery. Journal of the American College of Cardiology, 52, $117-123$.

Liu, A., Chen, Y., Yang, Z., Feng, Y., Rui, W., Luo, W., et al. (2009). New metabolites of fenofibrate in Sprague-Dawley rats by UPLC-ESI-QTOF-MS-based metabolomics coupled with LCMS/MS. Xenobiotica, 39, 345-354.

Malinowski, K., Christensen, R. A., Konopka, A., Scanes, C. G., \& Hafs, H. D. (1997). Feed intake, body weight, body condition score, musculation, and immunocompetence in aged mares given equine somatotropin. Journal of Animal Science, 75, $755-760$

McDougall, G., Martinussen, I., \& Stewart, D. (2008). Towards fruitful metabolomics: High thoughput analyses of polyphenol composition in berries using direct infusion mass spectrometry. Journal of Chromatography B, 871, 362-369.
Nicholson, J. K., Connelly, J., Lindon, J. C., \& Holmes, E. E. (2002). Metabonomics: A platform for studying drug toxicity and gene function. Nature Review Drug Discovery, 1, 153-162.

Nicholson, J. K., Lindon, J. C., \& Holmes, E. E. (1999). Metabonomics: Understanding the metabolic responses of living systems to pathophysiological stimuli via multivariate statistical analysis of biologica NMR spectroscopic data. Xenobiotica, 29, 1181-1189.

Platt, J., Uy, O., Heller, D. N., Cotter, R. J., \& Fenselau, C. (1988). Computer-based linear regression analysis of desorption mass spectra of microorganisms. Analytical Chemistry, 60, 1415-1419.

Popot, M. A., Bobin, S., Bonnaire, Y., Delahaut, P. H., \& Closset, J. (2001). IGF-I plasma concentrations in non-treated horses and horses administered with methionyl equine somatotropin. Research in Veterinary Science, 71, 167-173.

Rahmanian, M. S., Thompson, D. L., \& Melrose, P. A. (1997). Immunocytochemical localization of prolactin and growth hormone in the equine pituitary. Journal of Animal Science, 75, 3010-3018.

Rijk, J. C. W., Lommen, A., Essers, M. L., Groot, M. J., Van Hende, J. M., Doeswijk, T. G., et al. (2009). Metabolomics Approach to Anabolic Steroid Urine Profiling of Bovines Treated with Prohormones. Analytical Chemistry, 81, 6879-6888.

Robertson, D. G. (2005). Metabonomics in toxicology: A review. Toxicology Sciences, 85, 809-822.

Sleighter, R. L., \& Hatcher, P. G. (2007). The application of electrospray ionization coupled to ultrahigh resolution mass spectrometry for the molecular characterization of natural organic matter. Journal of Mass Spectrometry, 42, 559-574.

Smith, C. A., Want, E. J., Tong, G. C., Saghatelian, A., Cravatt, B. F., Abagyan, R., et al. (2006). XCMS: Processing mass spectrometry data for metabolite profiling using nonlinear peak alignment, matching, and identification. Analytical Chemistry, 78, 779-787.

Szeszycki, E. E., Puckett, A. P., \& Ziegler, T. (1996). Les effets métaboliques de l'hormone de croissance. Nutrition Clinique Metabolisme, 1996(10), 311-323.

Theodoris, G., Gika, H. G., \& Wilson, I. D. (2008). LC-MS-based methodology for global metabolite profiling in metabonomics/ metabolomics. Trends in Analytical Chemistry, 27, 251-260.

Thompson, D. L., Rahmanian, M. S., Depew, C. L., Burleigh, D. W., Desouza, C. J., \& Colborn, D. J. (1992). Growth hormone in mares and stallions: Pulsatile secretion, response to growth hormone-releasing, hormone, and effects of exercise, sexual stimulation, and pharmacological agents. Journal of Animal Science, 70, 1201-1207.

Tolstikov, V. V., \& Fiehn, O. (2002). Analysis of highly polar compounds of plant origin: Combination of hydrophilic interaction chromatography and electrospray ion trap mass spectrometry. Analytical Biochemistry, 301, 298-307.

Tolstikov, V. V., Lommen, A., Nakanishi, K., Tanaka, N., \& Fiehn, O. (2003). Monolithic silica-based capillary reversed-phase liquid chromatography/electrospray mass spectrometry for plant metabolomics. Analytical Chemistry, 75, 6737-6740.

Warrack, B. M., Hnatyskyn, S., Ott, K. H., Reily, M. D., Sandors, M., Zhang, H., et al. (2009). Normalization strategies for metabonomic analysis of urine samples. Journal of Chromatography $B$, $877,547-552$. 